\title{
NOMBRES PROPIOS
}

En este trabajo pretendemos, en realidad, examinar sólo uno de los muchos problemas que plantean los nombres propios: su relación con ciertas expresiones descriptivas $y$, sobre todo, la manera cómo éstas deben concebirse. Nuestras conclusiones son limitadas e intentan preparar el terreno para una discusión más amplia.

En el tratado $A$ System of Logic ${ }^{1}$ Stuart Mill utiliza la distinción entre nombres 'connotativos' y nombres 'no-connotativos'. Ejemplo de lo primero serían, según el lenguaje de ese autor, aquellas palabras que denotan a un sujeto e implican un atributo. Así, 'blanco', 'largo', 'rojo', 'virtuoso' son connotativas; la palabra 'blanco', para tomar una entre tantas, denota a todas las cosas blancas, la nieve, la hoja de papel, la espuma del mar y connota el predicado blancura. Conviene recordar, por otra parte, que todas las palabras que Stuart Mill llama 'generales concretas' son connotativas; 'Hombre', digamos, denota a Pedro, a Juan, a Rafael y a un número indefinido de individuos, connotando unos ciertos predicados. Debe quedar claro, entonces, que los términos connotativos no denotan a las propiedades o características que definen la pertenencia a una determinada clase, sino que denotan a los miembros de la clase e implican - connotan- ciertos predicados cuya posesión por parte del individuo permite incluirlos dentro de una clase dada. Cuando de algún individuo, por tanto, afirmamos que es un hombre, 'Hombre' denota a la persona en cuestión y no a los predicados que constituyen su humanidad. Dicho con otras palabras, incluimos a un individuo dentro de una clase basándonos en ciertas propiedades suyas. Esto significa que cuando aplicamos una palabra del tipo de 'hombre', lo hacemos porque o en virtud de que los individuos poseen ciertas propiedades. Ésta seria, pues, una primera condición - Condición I- para clasificar una palabra como connotativa: que el uso correcto de la palabra sólo puede llevarse a cabo en virtud de, o tomando en cuenta, ciertas propiedades del objeto o individuo. Esto es: una expresión es connotativa cuando es parafraseable por predicados o expresiones predicativas.

Pero esta primera condición no sólo la cumplen palabras como 'Hombre', 'Hombre Varón', 'Silla', etc., etc., sino también las llamadas descripciones definidas. En efecto, una descripción definida se aplica a un objeto $o$ individuo precisamente porque tiene tales o cuales propiedades. En ambos casos la aplicación sería unívoca, en virtud de las mismas propiedades y, por consiguiente, los predicados que las parafrasearian serían los mismos. Y, na-

1 J. Stuart Mill, A System of Logic, Book I, ch. II. 
turalmente, también en ambos casos los predicados que entran en juego tienen una aplicación general. Detengámonos aquí con el objeto de evitar algunas confusiones. Por ejemplo, la que consistiría en pensar que dado que las descripciones definidas pretenden referirse a un solo individuo, ello nos comprometería a aceptar la idea, posiblemente contradictoria, de predicados que no sólo por razones contingentes están limitados a un único objeto -lo cual es perfectamente compatible con la afirmación de su generalidad- sino de predicados que no pudieran, por alguna razón de principio, haberse aplicado a ningún otro individuo. Se apelaría a ciertas descripciones definidas que por la naturaleza de su contenido, o aspecto enunciativo, sólo podrían emplearse para referirse a un individuo. Un caso entre muchos sería el siguiente: "El primer hombre que caminó en la Luna"; aquí es evidente que no puede haber dos individuos en el universo que satisfagan a la descripción definida. Podría parecer, entonces, que este tipo de expresiones, cuya connotación nadie disputa, nos obligaría a asumir predicados con una extensión limitada en principio. Pero obsérvese, a fin de alejar este peligro, que las expresiones predicativas que componen a la descripción definida - "primer hombre", "caminar en la Luna"- de ninguna manera están limitadas a un único individuo. Por consiguiente, una cosa es decir que no puede haber más de un individuo del cual se predique una determinada conjunción de propiedades o predicados y otra cosa muy distinta es decir que esos predicados sólo pueden afirmarse de un individuo. Lo primero es claramente plausible y es, en verdad, lo que ocurre en "El primer hombre que caminó e n alLuna"; lo segundo probablemente es incoherente. Predicados no-limitados nos permiten referirnos al único individuo que los satisface. Así, pues, sería una conclusión errónea creer que si hay un solo individuo que satisface ciertos predicados, éstos deben concebirse como limitados, por principio, a ese individuo. Con mínimas variantes, lo mismo podría decirse de otros ejemplos, tales como "El hombre que inventó la penicilina". Aquí parecería que el predicado "inventar la penicilina", a diferencia de "caminar en la Luna", está limitado, por fuerza, a un solo individuo. Por lo pronto cabe observar lo mismo que en el caso anterior: en la expresión predicativa "inventar la penicilina" se encuentra a su vez un predicado - "ser inventor"- cuya generalidad está fuera de toda duda. Pero, además, debemos notar lo siguiente: es verdad que "inventar la penicilina" en rigor se predica de una sola persona y también es verdad que su extensión está limitada, por tanto, a un solo miembro; pero de ninguna manera es un predicado que no pudiera haberse aplicado a otros individuos, porque . al fin y al cabo es una verdad contingente que Fulano de Tal haya inventado algo. Antes de que se hubiese fabricado la medicina en cuestión, el predicado "inventar la penicilina" estaba, por así decirlo, en disposición de aplicarse a cualquier individuo y no estaba destinado a uno en particular. 
Una cosa es, entonces, reconocer que el predicado se aplica de facto a un solo y determinado individuo y otra es sostener que sólo podría haberse aplicado a ese individuo; una cosa es que la extensión conste de un solo miembro y otra decir que la extensión debia limitarse a ese miembro. Por otra parte, conviene mencionar, aunque sea brevemente, otro problema relacionado con lo anterior. Prima facie hay sin duda una diferencia entre "El primer hombre que caminó en la Luna" y descripciones definidas del tipo "El actual Rey de Francia" - para volver a recoger esta cansada frase de la filosofía contemporánea. Coinciden en su intento de referirse a un solo individuo y en los dos casos las proposiciones en las que figuren como sujetos gramaticales resultarian falsas si hubiese más de un individuo que las satisficiese. Sin embargo, descripciones como "El actual Rey de Francia", "El actual Presidente de Venezuela", etc., pueden ser usadas en diferentes ocasiones para referirse a diferentes individuos - aunque claro está que en cada una de las ocasiones no debe haber más de un individuo- en tanto que "El primer hombre que caminó en la Luna" sólo podría usarse para referirse siempre al mismo individuo.

Ahora bien, si se sostiene que las expresiones connotativas se predican de los individuos unívocamente, entonces resultará claro que la Condición 1 explicita condiciones necesarias, pero no suficientes. En efecto, la Condición I afirma, únicamente, que si son connotativas se aplican en virtud de propiedades, pero no establece que sean las mismas, que la predicación sea unívoca. De manera que si estas palabras se consideran como paradigma de lo connotativo, es necesario hacerle justicia a esa otra característica. Pero que la predicación sea unívoca quiere decir que se fijan de una manera clara las propiedades que justificarán la aplicación de ia palabra, esto es, se da una definición del término o términos en cuestión. La consecuencia inmediata es la siguiente: que dichas expresiones se usarán correctamente cuando el objeto o individuo tenga unas propiedades específicas y no podrá usarse la palabra cuando carezca de ellas. Si, no obstante, se aplica, se habrá predicado algo falso del individuo. Lo anterior es equivalente a decir que la relación entre expresiones connotativas y ciertos predicados es analítica. Ésta sería la segunda condición - Condición II- para decidir si una palabra es o no es connotativa. Quede claro que esta condición excluye como posible connotación de la palabra a todas las propiedades del individuo que no formen parte de su definición. Por último, conviene tener presente que a veces se utiliza una tercera condición, la que exige que las palabras connotativas "informen" $o$, de manera más suelta, "digan" algo. La terminología es vaga aunque en todo caso la idea general pretende apoyarse en una verdad trivial: si un término connota, se refiere describiendo y, entonces, afirma ciertos predicados del objeto o del individuo. En este sentido, la tercera condición es un corolario de las anteriores y, en rigor, suele considerársela 
como una consecuencia de la segunda. Lo que se entiende por información' es, entonces, la que se deriva de los predicados que están analíticamente unidos a la palabra, excluyéndose cualquier otro tipo de información que, obviamente, puede ser muy rica y variada, emotiva, estilística, etc., etc. Por el mismo razonamiento, si las palabras 'informan' en este último sentido, no por ello se concluye que connotan en la acepción semánticamente rígida de la Condición II.

La pregunta es, ahora, la siguiente ¿cuál es la situación de los nombres propios frente a las dos condiciones propuestas? La respuesta es algo más compleja de lo que quizá pudiera pensarse. Con el propósito de esquematizar y levantar un mapa un poco borroso de esta zona conceptual, vamos a dividir las posiciones en estos apartados:

I) Los que se apoyan en la Condición I y concluyen que los nombres propios son connotativos. Un ejemplo sería B. Russell cuando sostiene que son descripciones encubiertas o disfrazadas. ${ }^{2}$

2) Los que, simplemente, se apoyan en la Condición II para negar que los nombres propios sean connotativos. Stuart Mill sería uno de Ios representantes de esta postura. ${ }^{3}$

3) Los que han investigado las relaciones que guarda el nombre propio con las expresiones predicativas relativas a un objeto $o$ individuo. -Debe mencionarse a L. Wittgenstein, a P. F. Strawson y a J. R. Searle.4 Sobre la posición de este último autor volveremos más adelante. Lo que haremos a continuación es recorrer algunos de estos caminos.

Por lo pronto debemos establecer cuál sería la connotación adecuada a los nombres propios, esto es, de qué tipo serían, en el supuesto caso de que los nombres propios satisficieran las condiciones I y II, los predicados o las expresiones predicativas que estarian unidos a ellos, ya sea analíticamente o de otro modo. De inmediato cabe observar que si la connotación de un nombre propio se concibe como constituida por términos que se predican de muchos o diversos individuos u objetos - como sucede con 'Silla', 'Hombre', 'Rinoceronte', etc., etc., y cuya connotación vamos a llamar general-, se dejaría quizá sin explicar la función más importante —aunque no exclu-

2 Véase, entre otros, The Philosophy of Logical Atomism, VI, recogido en Logic and Knowledge, Allen and Unwin, 1956, pp. 241 ss.

3 Op. cit., Book I, ch. II.

4 L. Wittgenstein, Philosophische Untersuchungen. B. Blackwell, 1958, parágrafos 4079; P. F. Strawson, Proper Names, The Aristotelian Society, Supplementary Volume, XXXI, 1957; J. R. Searle, "Proper Names", artículo recogido en Philosophical Logic, O. U. P., 1967, y el artículo "Proper Names and Descriptions" que se encuentra en The Encyclopedia of Philosophy, Vol. V1. The Macmillan Company and the Free Press, New York, 1967 . 
siva - de estas palabras, a saber, la referencia a individuos particulares. Para evitar una posible confusión, no exenta de complicaciones, conviene recordar que los "nombres generales" - para seguir con la terminología tradicionalse aplican desde luego a individuos, pero no se refieren específicamente a ninguno de ellos. Así, "Hombre" u "Hombre Varón" puede aplicarse a Pedro, Juan y Lorenzo, aunque no se refieren en particular a ninguno de ellos, que es, en cambio, la tarea que llevan a cabo los nombres propios, las descripciones definidas y los llamados particulares egocéntricos. De manera que si la connotación de un nombre propio es general, habría que abandonar la tesis, admitida por todos sin mayores discusiones, de que los propios son nombres 'singulares', como los bautiza Stuart Mill. La conclusión es la siguiente: la posible connotación de un nombre propio debe ser la conveniente o adecuada a su función de indicador individual. Esto es, cuando se introduce el término 'connotación' se supone que es para dar una explicación del uso de la palabra en cuestión; de modo que cuando se propone un esquema de connotación, la primera condición que debe satisfacerse es la de que haya una adecuación entre aquél y el empleo efectivo del término. Claro está que el cumplimiento de esa condición no es suficiente para que un esquema sea correcto; sin embargo, si se cumple la condición mencionada se garantiza, cuando menos, que la discusión sea relevante y apropiada. Por consiguiente podremos descalificar, sin consideraciones ulteriores, cualquier tesis que propusiera como connotación del nombre propio una general; quien insistiera en ella estaría abandonando o alterando la función que se le había asignado a 'connotación'. La discusión, por el contrario, debe plantearse al nivel de expresiones predicativas que describan, pero que a la vez individualicen, a un objeto particular específico; es decir, expresiones predicativas complejas que permitan referirse a un individuo u objeto determinado, que es - no lo olvidemos- lo que hacen los nombres propios. "Hombre Varón" es una expresión, claro está, que permite describir a un individuo, pero que no es capaz, por sí sola, de individualizarlo - por lo menos si atendemos a su uso paradigmático. Por tanto, la discusión acerca de si un nombre propio tiene o no connotación se presenta como equivalente a la discusión sobre las relaciones que guarda el uso correcto de un nombre propio con ciertas descripciones definidas. Éste es el ámbito del problema. Es necesario, sin embargo, hacer una aclaración. Se trata de lo siguiente. Consideremos tres tipos diferentes de descripciones: (A) "El actual Rey de Francia"; (B) "El primer hombre que caminó en la Luna"; (C) "El hombre que ayer fue al cine Rex". Con respecto a lo que ahora nos interesa, cada tipo presenta, respectivamente, estas características: 
(A) I) el reiterado uso de la expresión, en el sentido señalado páginas atrás, a saber de que es posible usarla en diferentes circunstancias para referirse a individuos diferentes. 'Reiterado uso' no quiere decir, entonces, proferir o escribir la expresión muchas veces, ni tampoco emplearla muchas veces para referirse al mismo individuo.

2) en cada caso en que se usa se refiere a un individuo en particular.

3) de dicho individuo se da una descripción única o singular, en el sentido de que es

4) satisfecha por un solo individuo.

(B) I) no tiene un uso reiterado.

2) se refiere a un individuo en particular.

3) de dicho individuo se da una descripción única o singular, en el sentido de que es

4) satisfecha por un solo individuo.

(C) 1) tiene reiterado uso

2) en cada caso en que se usa se refiere a un individuo en particular.

3) de dicho individuo no se da una descripción única o singular,

4) la descripción es satisfecha por muchos individuos.

De lo anterior se derivan, tal vez, unas cuantas enseñanzas. En primer lugar, vemos que es perfectamente compatible que una expresión pueda usarse para referirse a un individuo aun cuando haya muchos que puedan satisfacerla. Pero, en cambio, sí se requiere que haya un solo individuo que la satisfaga si la descripción, además de referirse a un particular, es individualizante. Por descripción individualizante entenderemos, entonces, aquellas que cuando se usan, esto es, en el momento de su uso, no toleran que haya más de un individuo que las satisfaga. Así, las descripciones del tipo (A) y (B) son individualizantes. Las del tipo (C), por el contrario se comportan de otra manera. Nadie dejaría de describir a un individuo como "El muchacho del sweater rojo" porque tenga pruebas de que hay otros muchachos con un sweater de ese color. Las descripciones del tipo (C) toleran la pluralidad, no son individualizantes. Lo que sí puede ocurrir es que se den situaciones en las que haya un solo inclividuo al cual pueda aplicársele una descripción no-individualizante; en esos casos una descripción del tipo (C) puede funcionar como una descripción identificante -en ese contexto. "El muchacho del sweater rojo" puede ser usada para distinguir a un individuo si se la emplea en un grupo en que solamente uno de ellos lleva sweater rojo. Pero esto, claro está, no borra la distinción anterior.

El problema es, ahora, fijar de qué tipo deben ser las descripciones que se tomen en cuenta en la discusión acerca de la connotación de los nombres 
propios. Para ello es menester, primero, reflexionar sobre la forma en que las descripciones intervienen en el uso de los nombres propios. Cuando un nombre propio se usa correctamente, entre otros pueden discernirse los siguientes elementos: I) la referencia a un individuo determinado; 2) la aplicación del mismo nombre propio al mismo individuo en diferentes circunstancias. Esto significa que para usar un nombre debidamente es necesario saber cuál es el individuo que lleva ese nombre y este conocimiento no puede constituirse más que sobre la base de propiedades del individuo. Ésta es una condición o un prerrequisito. De donde se deriva una exigencia cuando menos teórica: que quien usa correctamente un nombre propio debe, en principio, ser capaz de describir, de suministrar, alguna descripción del individuo en cuestión, pues el nombre propio no 'dice' a quién se está refiriendo y, además, puede serlo de diferentes individuos. La plausibilidad de esta exigencia salta a la vista cuando advertimos lo extraño que serfa que una persona empleara un nombre propio y, sin embargo, no supiera absolutamente nada del individuo que lleva ese nombre; sería extraño porque ello equivaldría a ignorar a quién nombra la palabra, cuál es el individuo al que se refiere. No podría, en suma, estar usando el nombre. Dijimos que esta exigencia es teórica, porque no supone que, en la práctica, quien usa un nombre propio correctamente siempre es capaz de dar una descripción verbal del individuo; ${ }^{5}$ pero en todo caso el usuario debe poder individualizar y esto, nuevamente, sólo es posible basándose en propiedades del individuo, lo cual es una especie de equivalente preverbal de las descripciones. Tampoco se sigue que cuando se emplea un nombre propio el usuario deba tener presente una determinada descripción que le permitiría, a continuación, aplicar el nombre. No se sigue, porque no se están describiendo aqui mecanismos psicológicos - por otra parte improbables-, sino que se establecen condiciones semánticas del uso de los nombres propios. Otra manera de indicar la relación estrecha que guarda un nombre propio con las descripciones es observando cómo se enseña y se aprende el uso de este género de palabras. ${ }^{6}$ Parece, por tanto, como si el empleo de cualquier nombre propio obligara a conectarlo con descripciones del individuo; se cumpliría entonces la Condición I. Ahora ya estamos en posibilidad de responder cuál es el tipo de las descripciones que intervienen en el uso del nombre propio: deben ser el tipo (A) y (B), esto es, descripciones individualizantes. En efecto, si para usar correctamente un nombre propio es necesario conectarlo con un individuo en particular, entonces es casi un truismo agregar que las descripciones de dicho individuo deben aplicarse únicamente a él, pues de lo contrario no tendrían la fuerza suficiente para distinguir

5 Piénsese en un niño pequeño que llama, digamos, a su hermano por su nombre y que aún no sabe construir oraciones.

6 Véase Searle, "Proper Names", p. 91; "Proper Names and Descriptions", p. 490. 
a ese individuo. Nuevamente conviene recalcar que en una situación pragmática una descripción no-individualizante - sino del tipo (C) - puede cumplir, según se puntualizó, una función identificante. Si alguien habla de un tal Juan y preguntamos quién es esa persona, una respuesta perfectamente en orden es la que dice que se trata de "El muchacho del sweater rojo" - perfectamente adecuada si no hay ninguna otra persona con esa ropa. ${ }^{7}$ Teóricamente, aunque en la práctica no necesariamente, las descripciones individualizantes son las que están en relación con el uso de los nombres propios.

Ahora bien, si se cumple la Condición I, esto significa que en cualquier uso determinado de un nombre propio, éste es parafraseable por descripciones del individuo al cual se refiere. Con otras palabras, es sustituible por descripciones. Si un niño encuentra en un libro la mención de 'Julio César' y nos pregunta quién es ese individuo $\multimap$ si ya está infectado de semántica, a quién se refiere ese nombre - procederemos a dar una serie de descripciones del general romano. Por otro lado hay que agregar que el contexto guia, por así decirlo, la elección de las descripciones que van a sustituir al nombre propio. Si, por ejemplo, estoy hablando de Caldera y uno de mis oyentes me hace saber que ignora a quién me refiero, lo más probable es que sustituya el nombre por la descripción "El actual Presidente de Venezuela" y no por "El autor del libro Andrés Bello publicado en Caracas en 1946". Pero las exigencias del contexto pueden ser todavía más severas. Detengámonos aquí un momento. Supongamos que en una conversación sobre política hispanoamericana afirmo que las ideas de Caldera con respecto a las compañías petroleras son indecisas o que no ofrecen novedad alguna; que Caldera ha hecho públicos sus deseos de que todos los partidos de izquierda trabajen dentro de la legalidad; que Caldera apoya la tesis de que Venezuela tenga relaciones diplomáticas con todos los países del mundo, etc., etc. Si después de estas declaraciones alguien me preguntara a quién me refiero con ese nombre, sin duda me inclinaría a responder, también en esta ocasión, que se trata del actual Presidente de Venezuela. Pero lo interesante es notar que el contexto de discurso casi obliga a dar esa respuesta u otra equivalente; es decir, el contexto guia - en este ejemplo en una forma menos vacilante- las descripciones que se eligen. Si yo hubiese contestado que Caldera era el autor de tal libro, mi descripción hubiera sido teóricamente apropiada, pero irrelevante en esa secuencia de discurso. El discurso selecciona la descripción o descripciones del individuo que 'juegan' con lo que se está afirmando. De manera que si, por una parte, los nombres propios son sustituibles por des-

7 Habría que investigar las relaciones entre las descripciones individualizantes y la función identificadora. Lo cual lleva al tema, muy complejo, de las relaciones entre descripciones individualizantes y egocentricidad. Véase Proper Names de Strawson y, sobre todo, su libro Individuals, Methuen, London, 1959. 
cripciones y, si por otra, el contexto de discurso crea una cierta selección entre ellas, hay alguna plausibilidad en la tesis que sostiene que los nombres propios funcionan en sus usos concretos -léase, en 'contextos concretos'- como descripciones encubiertas. Así, pues, la tesis de que los nombres propios deben interpretarse como descripciones disfrazadas, la formularíamos de la siguiente manera: un nombre propio es, en general, sustituible por descripciones y un nombre propio se usa casi siempre en contextos específicos, lo cual significa que en ellos es sustituible por ciertas descripciones. El nombre propio está en lugar de esas descripciones. La perspectiva pragmática volvería justificable el concepto de 'descripción encubierta'. Naturalmente que sostener lo anterior es algo muy cercano a decir que los nombres propios son connotativos, pues nadie niega que las descripciones que ellos 'encubren' lo son. Sin embargo las cosas no pueden dejarse así. En verdad, nos parece a nosotros que las exigencias de relevancia contextual guían la elección de las descripciones, pero lo importante es saber cuál es la relación lógica que guardan con los nombres propios. Si Rafael Caldera no fuera el actual Presidente de Venezuela, no por ello habría usado mal el nombre; si hubiera empleado otra descripción - digamos, "El jefe del partido Socialcristiano de Venezuela" para seguir a tono con el contexto- y me hubiesen demostrado que estoy equivocado, tampoco se sigue que habría usado mal el nombre propio. En ambos casos simplemente habría afirmado una falsedad de Caldera. Los ejemplos podrian multiplicarse y variarse, pero la moraleja semántica sería la misma: el contexto nos dirige en la elección de las descripciones, pero ninguna de ellas es necesaria para usarlo. Lo que sí sucede, claro está, es que si ninguna de las descripciones que exige la relevancia del contexto se aplica a un individuo -en este caso a Caldera-, entonces la mención de sus opiniones en una discusión sobre política hispanoamericana - $y$, por tanto, la mención de su nombre- está totalmente 'fuera de lugar'. Es decir, la aplicación de ciertas descripciones es "necesaria" -entre comillas- a los efectos de la relevancia, pero no es condición necesaria para aplicar el nombre. Por otro lado, las descripciones exigidas por la relevancia tampoco son "ésta y aquélla" sino, más bien, "ésta o aquélla": no creemos, en suma, que haya una "necesidad" de la relevancia en lo que se refiere a alguna descripción. Pero si la hubiera, debe distinguirse pulcramente de la otra necesidad. En conclusión, la "sustitución contextual" no prueba necesidad en ninguno de los dos sentidos. Ahora vemos, sin embargo, que si traducimos el concepto de 'descripción encubierta' al concepto de 'descrípciones relevantes' entonces podremos admitir su aplicabilidad y utilidad.

La situación en la que nos encontramos es la siguiente: el uso correcto de los nombres propios se ajusta a la Condición I, pero no hemos analizado, salvo alguna mención a propósito de los ejemplos anteriores, cuál es la re- 
lación, desde el punto de vista de la Condición II, entre un nombre propio y las descripciones individualizantes. En otros términos, ¿hay una relación analítica entre el nombre y alguna de las descripciones? ¿Hay una relación analítica entre el nombre y el conjunto de todas las descripciones individualizantes posibles acerca de una persona? Es obvio que para responder afirmativamente a la primera pregunta tendríamos que encontrar una descripción, o varias de ellas, tales que si no se aplicaran al individuo tampoco podríamos usar el nombre. En la literatura sobre el tema se han señalado repetidamente las consecuencias indeseables que se seguirían si la relación se interpreta como analítica; más aún, uno de los criterios para rechazar alguna tesis sobre este problema es ver si abierta o solapadamente admite que la relación entre nombre y descripciones es analítica. Recordemos, brevemente, algunas de las dificultades. Si la relación entre un nombre propio y una o varias descripciones individualizantes es analítica, resulta entonces que la negación de una proposición en la que el nombre figura como sujeto y la descripción figura como predicado, es contradictoria y no falsa. Lo cual es un absurdo porque, por otro lado, queremos decir que las descripciones individualizantes describen hechos contingentes de un individuo, esto es, que es concebible que a dicho individuo no se le aplicara esa descripción. Por otra parte, si eligiéramos como connotación a todas las descripciones individualizantes de una persona, a la dificultad anterior se le agregaría esta otra: la historia de un individuo estaría contenida en su nombre, escribir su biografía se convertiría en un largo ejercicio matemático, una inmensa deducción a partir de una única premisa, su nombre. Pero, además, seleccionar ciertas descripciones y colocarlas en una relación analítica es equivalente a definir al individuo. ¿Es ésta una empresa que tenga sentido? Si el hecho de que las descripciones individualizantes se apliquen a un individuo es un hecho contingente ¿no es absurda, acaso, la idea de elegir una de ellas y decidir que las demás son accidentales? ¿Cuáles serían los criterios para poder legislar de esa manera? ${ }^{8}$ Esta situación se refleja en lo que sucede en la práctica lingüística: allí no reconocemos, ni implícita ni explícitamente, el empleo de ninguna descripción individualizante que haga las veces de una definición. ${ }^{9}$ No usamos un nombre propio como si hubiera una descripción

8 A. J. Ayer, "Names and Descriptions" en The Concept of a Person, MacMillan, 1963, p. 142: "It is rather that there is nothing by which an individual is essentially identified. .."

9 Véase esta parte, espléndida, del parágrafo 79 de las Investigaciones filosóficas:

"Pero cuando afirmo algo acerca de Moisés — ¿estoy siempre dispuesto a sustituir 'Moisés' por alguna de estas descripciones? Quizá diga: Por 'Moisés' yo entiendo el hombre que hizo lo que la Biblia informa acerca de Moisés o que, cuando menos, hizo muchas de las cosas que allí se dicen. Pero ¿cuántas? ¿He decidido cuánto debe probarse falso para que abandone mi proposición como falsa? ¿Tiene para mí el nombre 'Moisés' un uso fijo y unívoco en todas las ocasiones? ¿No es el caso, más bien, de que yo tengo, por así decirlo, toda una serie de sostenes a mi disposición y de que estoy dispuesto a 
privilegiada, o sea, estamos dispuestos a admitir que cualquiera de ellas podría ser falsa con respecto a un determinado individuo, lo cual elimina, de paso, la tentación de considerar a la conjunción de todas las descripciones individualizantes como una definición del individuo en cuestión.

Tenemos, entonces, que los nombres propios necesitan apoyarse en descripciones, pero no buscando en ellas un definiens, sino para establecer contacto con un determinado individuo. De allí que cualquier descripción individualizante o grupo de ellas sirva para ese propósito. ¿Cómo expresar esta situación? Searle, siguiendo el camino abierto por Wittgenstein, lo hace de la siguiente manera: con respecto al uso de un nombre propio las descripcio. nes individualizantes forman una disyunción. ${ }^{10}$ Esta conceptuación le hace justicia al hecho básico de que podemos negar -sin incurrir en contradicción- cada una de las descripciones y resalta, a su vez, el otro hecho capital, a saber, la necesidad de que haya algunas descripciones que permitan precisar de cuál individuo una palabra es su nombre. Searle formula esto muy claramente: "Tiene sentido negar alguno de los miembros del conjunto de descripciones del portador del nombre, pero negarlas todas es eliminar las precondiciones para usar el nombre." 11 Pero para Searle esto es igual a decir que la disyunción de las descripciones de un individuo si está analíticamente unida al nombre propio. Por otra parte, agregamos, las descripciones que exige la relevancia contextual formarían, según los casos, disyunciones parciales de la primera disyunción y quizá podría hablarse de 'analiticidad contextual' de las disyunciones parciales con respecto al nombre en un determinado contexto. Acerca de esta formulación, básicamente correcta, quisiéramos hacer una observación. Según este esquema, si contáramos con una sola descripción individualizante, la relación del nombre propio con ella sería analítica. No lo es cuando hay más de una descripción individualizante; si la hipótesis de un objeto con una sola descripción posible es co-

apoyarme en uno si me quitan al otro y lo contrario? - Considera aún otro caso. Cuando digo ' $\mathrm{N}$ ha muerto' puede, por ejemplo, suceder lo siguiente con el significado del nombre ' $N$ ': Yo creo que vivió un hombre a quien (1) he visto aquí y allá, que (2) se veía de este y aquel modo (fotos), (3) que hizo esto y aquello, (4) que en la vida civil llevaba este nombre ' $N$ '. Si me preguntan lo que entiendo por ' $N$ ', enumeraré todo lo anterior o bien sólo algunas cosas, que serán diferentes según las ocasiones. Mi definición de ' $N$ ', entonces, tal vez sería ésta: 'el nombre respecto del cual todo esto es cierto'. ¿Pero si se prueba que algo de esto es falso? ¿Estaré dispuesto a declarar falsa la proposición ' $\mathrm{N}$ ha muerto'- aun cuando lo que resulta falso es algo que me parece secundario? Pero ¿dónde están los limites de lo accidental? $\mathrm{Si}$ en un caso semejante yo hubiera dado una definición, entonces estaria dispuesto a alterarla."

La versión castellana de las Investigaciones filosóficas, de la cual es parte este parágrafo, aparecerá próximamente en las ediciones del Instituto de Investigaciones Filosóficas de la UNAM. La traducción estuvo a cargo del autor del presente artículo.

10 Searle sólo habla de descripciones. Es una lástima que en sus escritos, que sin duda se cuentan entre los mejores, no se encuentre nada acerca de los diferentes tipos de descripciones.

11 Searle, artículo de la Encyclopiedia, p. 490. 
herente o no, es una cuestión a discutir. Sin embargo, en el caso en que hubiera una sola descripción, parece más o menos claro que no sería propiamente una definición, sino la única descripción posible. En esa circunstancia, la liga entre el nombre y la descripción sería de hecho analítica, pero la explicación de su analiticidad sería diferente a la que damos usualmente.

Pasemos, ahora, a la cuestión de si un nombre propio está o no analíticamente unido a un término general como "Hombre", "Hombre Varón", etc. A estas alturas de la exposición, este problema debe plantearse del siguiente modo: cómo la pregunta acerca de si todas las descripciones individualizantes relativas a una persona implican, explícita o implícitamente, un mismo término general. Esto es, las descripciones individualizantes forman, como ya se dijo, una disyunción; si todas ellas implican un mismo término general, entonces el uso correcto de un nombre propio también lo implicará. Si, por el contrario, hubiera algunas descripciones que fueran individualizantes de una persona sin que ellas mismas implicaran un determinado término y hubiera otras descripciones, también individualizantes de la misma persona, que sí lo implicaran, entonces el nombre propio no implicaría ese término general. ¿Qué debemos responder? La cuestión es interesante, sobre todo porque obliga a precisar el concepto de "descripción individualizante". En efecto, dada la acepción que hemos venido manejando $-\mathrm{y}$ que, en lo sustancial, creemos adecuada- no parece que quedaran excluidas descripciones que individualizan a una persona tomando en cuenta propiedades espacio-temporales y que utilizan términos tan generales como el de 'objeto'. Si esto se deja así, es evidente que, por ejemplo, "Hombre Varón" no está implicado por el nombre propio que se aplica a un determinado individuo varón. El significado de 'objeto' podrá interpretarse como una disyunción, uno de cuyos miembros es "Hombre Varón", pero esto no es suficiente para crear una situación analítica entre los dos términos. Por lo demás es evidente que algún término general siempre estará implicado, pero entonces deberá ser de una gran generalidad, 'objeto', 'ente', etc. - para que así pueda cubrir a todo tipo de descripción individualizante. Quedarían descartados términos generales de menos extensión, como "Hombre", "Hombre Varón",12 etc. Ahora bien, teóricamente lo anterior no obligaría a especificar más el concepto de 'descripción individualizante'. Sin embargo, desde un punto de vista pragmático, se presentan dificultades. En efecto, ¿qué sentido tiene decir que una persona sabe cuál es el individuo al que se refiere con un nombre propio si de dicho individuo sólo puede dar una serie de descripciones individualizantes que lo 'fijan' mediante predicados espacio-temporales y términos de una generalidad máxima como 'objeto', 'ente', etc.? El uso de un nombre propio, ¿no exige, acaso, un conocimiento, 
por así decirlo, 'predicativamente más específico'?13 Esto sí supondría una reflexión a fondo sobre el concepto de 'descripción individualizante'; mientras no se aclare aún más, muchas de las conclusiones sobre este problema permanecerán en un estado vacilante.

InSTITUTO DE INVESTIGACIONES Filosóficas

Alejandro Rossi

Universidad Nacional Autónoma de México

13 Véase, en Proper Names, p. 215, cómo la caracterización que nos ofrece Strawson de las descripciones no elude estas dificultades. 